\title{
Insulinoma presenting with post-prandial hypoglycaemia following fundoplication
}

\author{
Sarah Y Qian', Matthew J L Hare', Alan Pham² and Duncan J Topliss',3 \\ Departments of ${ }^{1}$ Endocrinology and Diabetes, ${ }^{2}$ Anatomical Pathology, The Alfred Hospital, Melbourne, Australia, and \\ 3Department of Medicine, Monash University, Melbourne, Australia
}

Correspondence should be addressed to D Topliss

Email

duncan.topliss@monash.edu

\section{Summary}

Insulinomas are rare neuroendocrine tumours that classically present with fasting hypoglycaemia. This case report discusses an uncommon and challenging case of insulinoma soon after upper gastrointestinal surgery. A 63-yearold man presented with 6 months of post-prandial hypoglycaemia beginning after a laparoscopic revision of Toupet fundoplication. Hyperinsulinaemic hypoglycaemia was confirmed during a spontaneous episode and in a mixed-meal test. Localisation studies including magnetic resonance imaging (MRI), endoscopic ultrasound (EUS) and gallium dotatate positron emission tomography ( ${ }^{68} \mathrm{G}$ a Dotatate PET) were consistent with a small insulinoma in the mid-body of the pancreas. The lesion was excised and histopathology was confirmed a localised well-differentiated neuroendocrine pancreatic neoplasm. There have been no significant episodes of hypoglycaemia since. This case highlights several key points. Insulinoma should be sought in proven post-prandial hyperinsulinaemic hypoglycaemia - even in the absence of fasting hypoglycaemia. The use of nuclear imaging targeting somatostatin and GLP1 receptors has improved accuracy of localisation. Despite these advances, accurate surgical resection can remain challenging.

\section{Learning points:}

- Hypoglycaemia is defined by Whipple's triad and can be provoked by fasting or mixed-meal tests.

- Although uncommon, insulinomas can present with post-prandial hypoglycaemia.

- In hypoglycaemia following gastrointestinal surgery (i.e. bariatric surgery or less commonly Nissen fundoplication) dumping syndrome or non-insulinoma pancreatogenous hypoglycaemia syndrome (NIPHS) should be considered.

- Improved imaging techniques including MRI, endoscopic ultrasound and functional nuclear medicine scans aid localisation of insulinomas.

- Despite advances in imaging and surgical techniques, accurate resection of insulinomas remains challenging.

\section{Background}

Insulinomas are rare neuroendocrine tumours with an incidence of 0.4 per 100000 patient years and a median age of presentation of 50 years $(1,2)$. Generally, these tumours are benign with less than $10 \%$ exhibiting malignant features (2). Insulinomas classically present with fasting hypoglycaemia; however, the clinical presentation can be variable and delayed diagnosis is common. Despite considerable advances in localisation imaging, accurate surgical resection can be challenging.

\section{Case presentation}

A 63-year-old man presented to the endocrinology unit for further investigation of symptomatic hypoglycaemia of approximately 6-month duration. These episodes first began 3 weeks after he underwent laparoscopic revision of a Toupet fundoplication for recurrent gastro-oesophageal reflux. He reported adrenergic symptoms of shaking and diaphoresis as well as neuroglycopaenic symptoms of confusion and poor concentration. Symptom onset was typically $1-2 \mathrm{~h}$ after meals. Generally, these episodes 
were mild to moderate in severity and managed by selfadministration of simple and complex carbohydrates. The patient was trying to prevent episodes by adopting a lowcarbohydrate diet and eating small quantities frequently.

The patient had the comorbidities of gastrooesophageal reflux disease and hiatus hernia for which he underwent laparoscopic Toupet fundoplication 6 years prior to this presentation. His other comorbidities were that of depression and hypertension for which he was on stable doses of an antidepressant and a single antihypertensive agent.

\section{Investigation}

Venepuncture was performed during a hypoglycaemic episode, and results indicated hyperinsulinaemic hypoglycaemia (glucose: $1.8 \mathrm{mmol} / \mathrm{L}$, insulin: $460 \mathrm{U} / \mathrm{L}$ and C-peptide: $6.0 \mathrm{nmol} / \mathrm{L})$. The patient used a continuous interstitial glucose monitor (Abbott FreeStyle Libre), which confirmed the near-daily occurrence of postprandial hypoglycaemic episodes. There were no episodes of fasting hypoglycaemia.

Earlier investigations included an oral glucose tolerance test and liquid mixed-meal test which both failed to provoke hypoglycaemia over $48 \mathrm{~h}$. A CT pancreas with contrast did not demonstrate a discrete mass. A gastric emptying study showed rapid gastric emptying with 30 -min retention of 70\% (normal $>70 \%$ ), 1-h retention of $25 \%$ (normal $30-90 \%$ ) and 2-h retention of $2 \%$ (normal $<60 \%)$. It was felt that these results were not diagnostic for dumping syndrome given these were unchanged from a study performed 1 year prior when there were no clinical symptoms of hypoglycaemia (1 h retention 23\%, $2 \mathrm{~h}$ retention $6 \%$ ).

Sulphonylureas were not detected in the serum. Circulating insulin antibodies were not found. A modified mixed-meal test was conducted using the patients' usual breakfast foods (muesli, yoghurt, orange juice). At $3 \mathrm{~h}$, hyperinsulinaemic hypoglycaemia was demonstrated (glucose: $1.9 \mathrm{mmol} / \mathrm{L}$, insulin: $21.4 \mathrm{U} / \mathrm{L}$, proinsulin: $>99.9 \mathrm{pmol} / \mathrm{L}$ and C-peptide: $2280 \mathrm{pmol} / \mathrm{L})$. A prolonged fasting study was conducted which failed to provoke hypoglycaemia, although this was terminated at $48 \mathrm{~h}$ as it was considered to be low yield to continue further once other investigation results became available.

Despite the absence of fasting hypoglycaemia, the findings were highly suggestive of excess endogenous insulin; therefore, localisation studies were performed to identify an insulinoma. Given the history of upper gastrointestinal surgery, other diagnoses considered included non-insulinoma pancreatogenous hypoglycaemia syndrome (NIPHS) and dumping syndrome.

The patient underwent a ${ }^{68} \mathrm{Ga}$ Dotatate PET that demonstrated a small focus of intense Dotatate uptake in the body of the pancreas in keeping with an insulinoma (Fig. 1). An MRI scan was concordant, showing an illdefined T2 hyperintense area of uptake $5 \mathrm{~mm}$ in diameter that did not enhance with contrast (Fig. 2). To assist with surgical planning, endoscopic ultrasonography (EUS) was performed, which again confirmed a $6 \mathrm{~mm}$ hypoechoic lesion in the mid-body of the pancreas consistent with a small insulinoma. The lesion was tattooed with dye injection (Fig. 3).

\section{Treatment}

The patient underwent an uncomplicated laparoscopic distal pancreatectomy removing $75 \mathrm{~mm}$ of distal pancreas. Intraoperative linear laparoscopic ultrasound was used to localise the lesion. Unfortunately, four days postoperatively, the patient developed recurrent symptomatic post-prandial hypoglycaemia. Histology of the resected pancreas showed tattoo pigment and normal pancreatic tissue, but no tumour was identified.

A repeat ${ }^{68} \mathrm{Ga}$-Dotatate scan confirmed unresected insulinoma, $12 \mathrm{~mm}$ medial to the resection margin, and the patient underwent a second operation. For technical reasons, the laparoscopic procedure was converted to an open distal pancreatectomy. No lesion was identified macroscopically and intraoperative frozen section was inconclusive and unable to provide histological confirmation. A further $60 \mathrm{~mm}$ pancreatic specimen was excised.

Histopathological evaluation showed a $5 \mathrm{~mm}$ welldifferentiated pancreatic endocrine tumour comprising cells with coarse nuclear chromatin and granular cytoplasm, in a typical nested and trabecular growth pattern. Ki67 immunoperoxidase staining showed a proliferative index of $5-10 \%$, consistent with a grade 2 lesion by WHO classification (3). Immunohistochemistry was positive for the presence of chromogranin and synaptophysin, but paradoxically negative for the presence of insulin (Fig. 4). There were no features of nesidioblastosis.

\section{Outcome and follow-up}

Pleasingly, 6 months following successful resection of insulinoma, the patient has not experienced any 


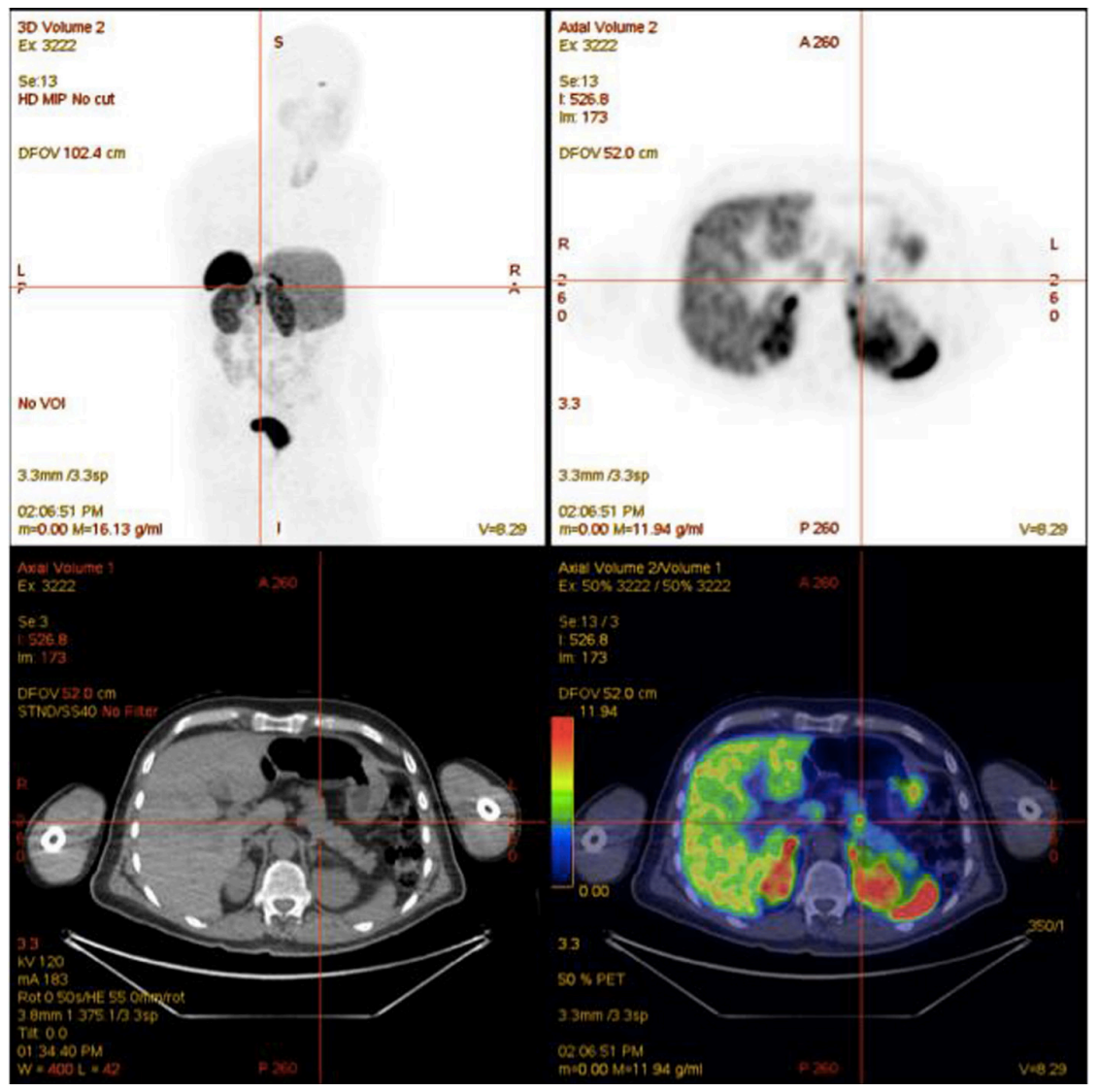

Figure 1

Ga68Dotatate PET demonstrating a small focus of intense uptake in the body of the pancreas in keeping with an insulinoma. No other abnormalities were detected elsewhere in the body. significant post-prandial hypoglycaemia. Of note, the patient reports mild post-prandial symptomatic hypoglycaemia with a lowest BGL of $3.4 \mathrm{mmol} / \mathrm{L}$. This may represent a degree of underlying dumping syndrome post upper gastrointestinal surgery, which supports our hypothesis of mixed pathology in this case.

\section{Discussion}

Insulinomas commonly present with hypoglycaemia characterised by Whipple's triad - sympathoadrenal and neuroglycopaenic symptoms of hypoglycaemia, blood glucose levels less than $3.0 \mathrm{mmol} / \mathrm{L}$ and resolution of symptoms following restoration of euglycaemia. Biochemical investigation can be performed in a spontaneous episode or one provoked by either 72-h fasting (fasting hypoglycaemia) or mixed-meal test (post-prandial hypoglycaemia). Endogenous hyperinsulinaemia is confirmed by elevated insulin greater than or equal to $3.0 \mathrm{U} / \mathrm{L}(18 \mathrm{pmol} / \mathrm{L})$, C-peptide greater than or equal to $0.6 \mathrm{ng} / \mathrm{mL}(0.2 \mathrm{mmol} / \mathrm{L})$, proinsulin greater than or equal to $5 \mathrm{pmol} / \mathrm{L}$, plasma $\beta$-hydroxybutyrate levels of $2.7 \mathrm{mmol} / \mathrm{L}$ or less and a plasma glucose concentration of greater than or equal to $25 \mathrm{mg} / \mathrm{dL}$ $(1.4 \mathrm{mmol} / \mathrm{L})$ following IV glucagon administration (4).
Fasting hypoglycaemia has long been regarded as a cardinal feature of insulinoma. However, there is growing recognition that either fasting or post-prandial

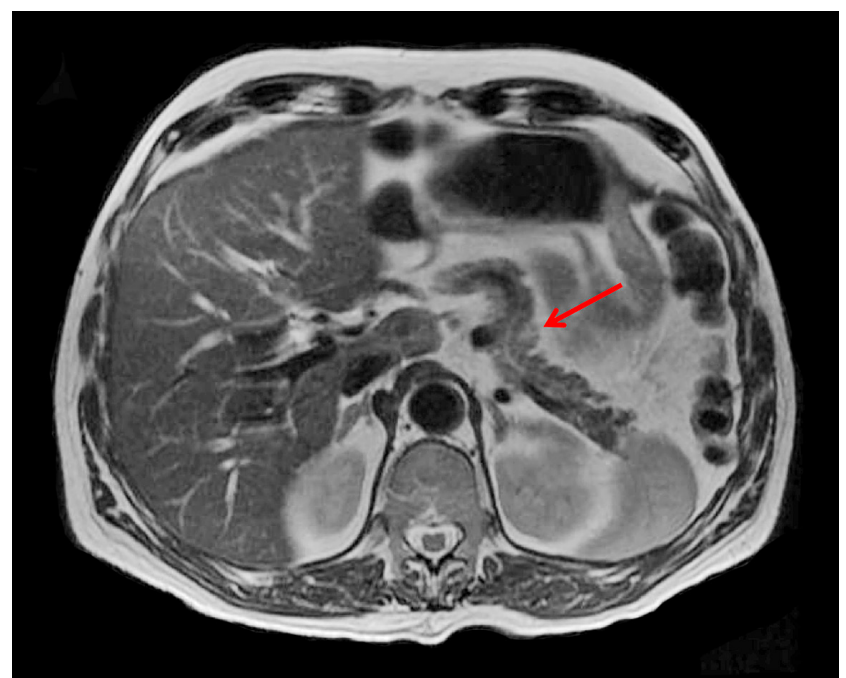

Figure 2

MRI pancreas T2-weighted image: subtle ill-defined T2 hyperintense lesion (red arrow) approximately $5 \mathrm{~mm}$ in diameter approximately $65 \mathrm{~mm}$ from the tip of the pancreatic tail. This lesion corresponds with the region of Dotatate uptake in the pancreatic body (Fig. 1). 


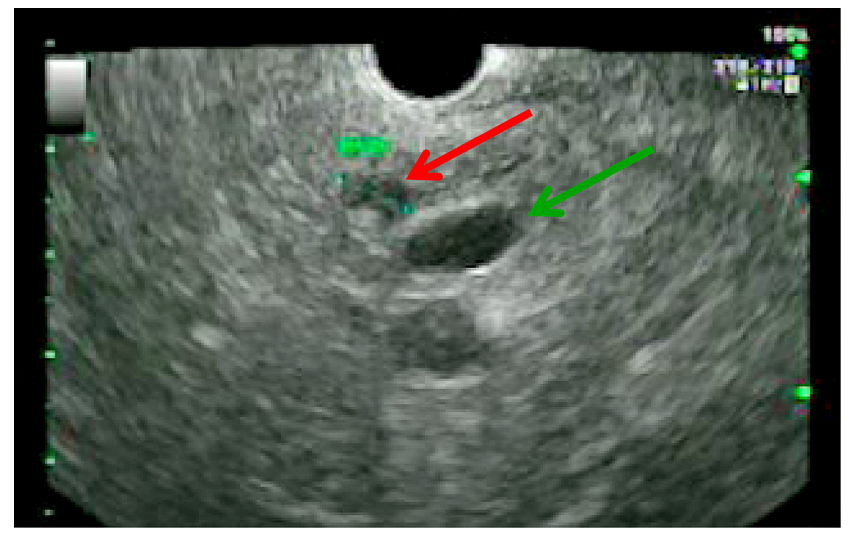

Figure 3

Endoscopic ultrasound: demonstrating a $6 \mathrm{~mm}$ hypoechoic lesion in the mid-body (red arrow) consistent with a small insulinoma, located within $5 \mathrm{~mm}$ of the main pancreatic duct (green arrow). No other abnormalities of the pancreas detected.

hypoglycaemia can occur (1). In a Mayo clinic series of 237 patients with insulinomas between 1927 and 2007, $6 \%$ presented with post-prandial hypoglycaemia only. Of these patients, 3 had negative 72-h fasting studies (1). These reports demonstrate the importance of pursuing localisation studies in patients with hyperinsulinaemic hypoglycaemia even in the absence of classical fasting hypoglycaemia.

The presentation of post-prandial hypoglycaemia following upper GI surgery may also relate to dumping syndrome, which occurs due to altered gastrointestinal anatomy resulting in large volumes of gastric content being delivered to the small bowel. In early dumping syndrome, symptoms occur about $45 \mathrm{~min}$ following a meal due to intravascular volume depletion due to osmotic fluid shift into the bowel lumen (5). A late form of dumping syndrome also occurs, presenting $2-4 \mathrm{~h}$ following a meal through an incretin-driven hyperinsulinaemic response triggered by the rapid delivery of carbohydrate to the duodenum (5).

A combination of early and late dumping syndrome can occur following GI surgery that disrupts or bypasses the pyloric sphincter. In the case of Nissen fundoplication, damage to the vagus nerve is thought to cause altered gastric motility leading to functional impairment. This is an acknowledged complication of Nissen fundoplication in children, but is also increasingly recognised in the adult population $(5,6)$.

Non-insulinoma pancreatogenous hypoglycaemia syndrome (NIPHS) is another cause for hyperinsulinaemic hypoglycaemia in patients with previous upper gastrointestinal surgery. This is a rare condition requiring
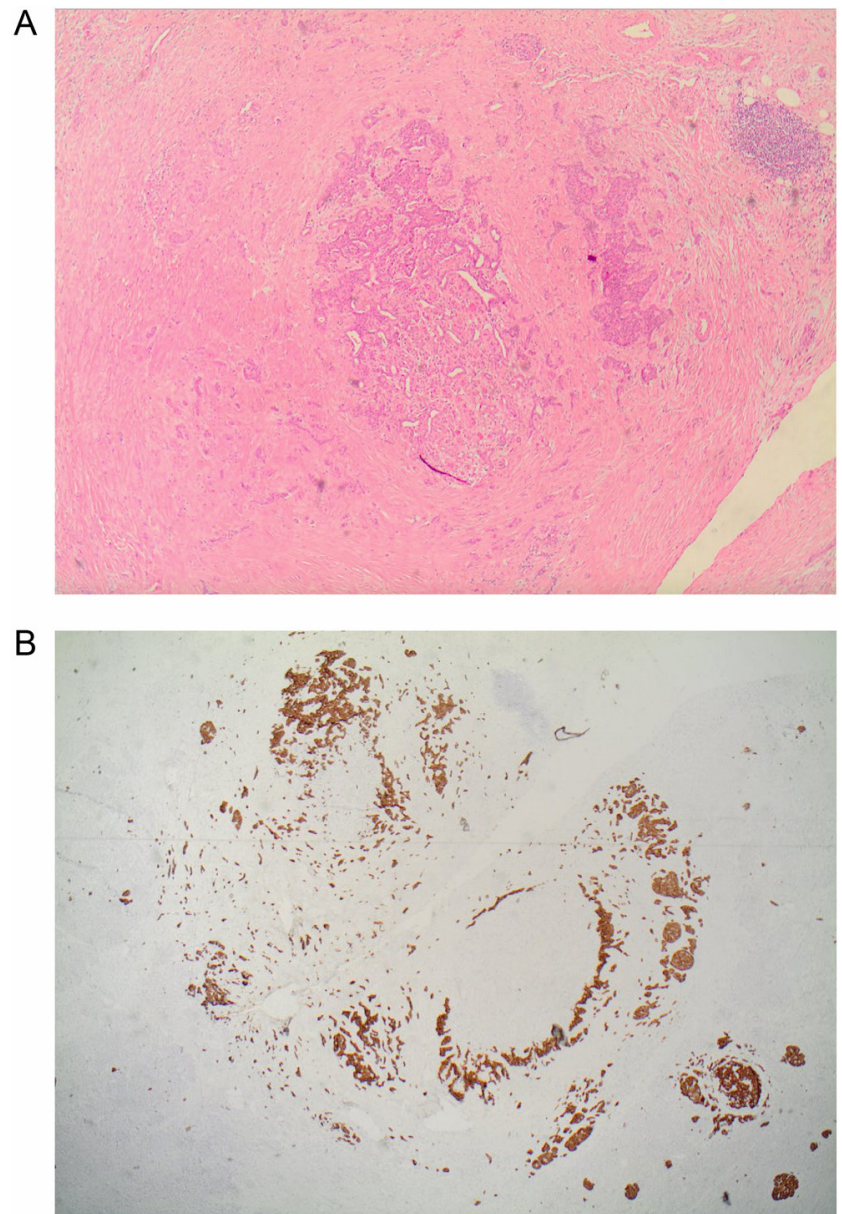

Figure 4

(A) Histopathology: Pancreatic tumour comprising a nested and trabecular proliferation of cells with a sclerotic stromal reaction (H\&E $\times 40)$. (B) Histopathology: Pancreatic tumour with a positive synaptophysin stain.

the following for diagnosis: positive Whipple's triad following a mixed-meal test in combination with a negative 72-h fast, negative localisation studies for insulinoma, positive arterial calcium stimulation test and histologically proven nesidioblastosis of pancreatic tissue. Pancreatic $\beta$-cell dysfunction occurs through hyperplasia and an altered incretin response. Upper GI surgery is thought to trigger this through altered GI architecture or by unmasking an underlying condition via weight loss and reduced insulin resistance (7). NIPHS is recognised post bariatric surgery, and a case has been reported following Nissen fundoplication (7). It is possible to speculate that revision of a gastric fundoplication could potentiate hypoglycaemia of an underlying insulinoma through an additional insult of NIPHS or dumping syndrome, but we cannot definitively diagnose or exclude this in our patient. 
Localisation studies have significantly improved in recent years. Traditional non-invasive imaging options include CT and MRI. On CT with contrast, an insulinoma is highly vascular with early-phase arterial contrast enhancement and venous washout. The sensitivity and specificity for CT diagnosis are $63-82 \%$ and $83-100 \%$ respectively (8). MRI findings typical of an insulinoma include low signal density on T1-weighted imaging, high signal density on T2-weighted imaging and early arterial phase contrast enhancement. The sensitivity and specificity of MRI diagnosis are $85-100 \%$ and $75-100 \%$, respectively (8).

Endoscopic ultrasound has further improved insulinoma detection and can localise lesions $2-3 \mathrm{~mm}$ in size (8). The sensitivity is dependent on tumour location, and detection in the pancreatic head and body is higher $(80-100 \%)$ than that in the pancreatic tail (37-60\%) (9).

Selective calcium arterial stimulation (SCAS) is an invasive modality involving the injection of calcium into the arteries supplying the pancreas. The stimulated insulin release is measured in the hepatic vein effluent and the distribution of response across the pancreas is assessed. While a focal response is suggestive of an insulinoma and a diffuse response supports nesidioblastosis, there are no established diagnostic criteria due to significant overlap between these pathologies (4). The role of SCAS in the diagnosis of insulinoma has diminished due to advances in nuclear medicine imaging.

Nuclear medicine imaging relies on radiolabelled detection of tumour receptor expression (somatostatin receptors, GLP1 receptors) or tumour metabolism (FDG, FDOPA) (10). Somatostatin receptors (SSR) have been used in octreotide scintigraphy and more recently in ${ }^{68} \mathrm{Ga}$ Dotatate PET/CT. However, while ${ }^{68} \mathrm{Ga}$ Dotatate PET/CT has improved sensitivity and specificity compared to octreotide scintigraphy, the use of SSR imaging is limited by its under-expression by tumour cells. SSTR type II is only expressed in $69 \%$ of insulinomas, and high-grade (G3) tumours are more likely to have low receptor density (10).

More recently, radiolabelled GLP1 analogues have been used as GLP1 receptors greater expression than SSTR in insulinomas (10). A case series of 40 patients with proven hypoglycaemia and inconclusive localisation studies (CT, MRI, EUS, somatostatin scintigraphy) demonstrated diagnostic benefit with the use of $99 \mathrm{mTC}$ GLP1 scintigraphy - 18 of 28 patients with positive 99mTc GLP1 scintigraphy had subsequent post-surgical histopathological confirmation of insulinoma (11).
Classical histopathological features of insulinomas include solid nest or trabecular cellular arrangement and cytological features of eosinophilic, granular cytoplasm and dispersed nuclei chromatin (12). Diagnostic immunohistochemical (IHC) findings include positive synaptophysin and chromogranin staining (12, 13). Functionality of neuroendocrine tumours is determined by the clinical presentation and does not reliably correlate with IHC staining for specific peptides, including insulin (12). In the described case, the diagnosis of pancreatic neuroendocrine tumour is confirmed by synaptophysin and chromogranin stain positivity, and while it is interesting that the insulin stain was negative, this unusual finding does not contraindicate the diagnosis. It is a previously described finding (14), perhaps due to rapid secretion and reduced cytoplasmic insulin storage.

Gastroenteropancreatic neuroendocrine neoplasms (GEP-NENs) are classified according to the revised WHO classifications (2010) as either well-differentiated (neuroendocrine tumour, NET) or poorly differentiated (neuroendocrine carcinomas, NEC) according to the Ki-67 proliferation index. NETs are further classified as G1 (Ki-67 $\leq 2 \%$ ) or G2 (Ki-67 3-20\%), and NECs are classified as G3 (Ki-67 >20\%) (3, 13). GEP-NENs can then be further staged according to TNM criteria based on tumour size, lymph node invasion and distant metastases (13).

Surgical resection remains the mainstay of treatment for insulinoma and should be considered even in metastatic disease (13). Laparoscopic surgical resection is associated with shorter hospitalisation compared to traditional open surgical approaches; however, a disadvantage is the inability to perform manual palpation for intraoperative localisation. Laparoscopic ultrasonography can be useful and has been shown to have a sensitivity of around $90 \%$ (15). As this case demonstrates, however, the resection of small insulinomas can be challenging despite the use of pre-operative and intraoperative localisation techniques.

\section{Conclusion}

This case demonstrates an uncommon presentation of insulinoma. Several features are non-classical including the absence of fasting hypoglycaemia and negative insulin staining on histopathology; however, these do not preclude the diagnosis of insulinoma as described in the literature $(2,14)$. Indeed, insulinoma remains an important diagnosis to exclude in isolated postprandial hyperinsulinaemic hypoglycaemia and further localisation studies including functional nuclear imaging should be considered. 
In the context of previous gastrointestinal surgery, other factors may also be contributing to the presentation of hypoglycaemia. For example, a form of dumping syndrome following gastric fundoplication unmasking hypoglycaemia from an underlying insulinoma cannot be excluded.

Finally, despite significant recent advances in the imaging and surgical treatment of insulinomas, successful management and cure remains challenging.

\section{Declaration of interest}

The authors declare that there is no conflict of interest that could be perceived as prejudicing the impartiality of the research reported.

\section{Funding}

This research did not receive any specific grant from any funding agency in the public, commercial or not-for-profit sector.

\section{Patient consent}

Written informed consent was obtained from the patient for the publication of this article.

\section{Author contribution statement}

S Y Qian: case management, case analysis for MS and writing the MS $60 \%$. M J L Hare: case management, case analysis for MS, 10\%. A Pham: case analysis (pathology) for MS, 5\%. D J Topliss: case management, case analysis for MS, writing the MS, $25 \%$.

\section{References}

1 Placzkowski KA, Vella A, Thompson GB, Grant CS, Reading CC, Charboneau JW, Andrews JC, Lloyd RV \& Service FJ. Secular trends in the presentation and management of functioning insulinoma at the Mayo Clinic, 1987-2007. Journal of Clinical Endocrinology and Metabolism 200994 1069-1073. (https://doi.org/10.1210/jc.20082031)

2 Service FJ, McMahon MM, O’Brien PC \& Ballard DJ. Functioning insulinoma - incidence, recurrence, and long-term survival of patients: a 60-year study. Mayo Clinic Proceedings 199166 711-719. (https://doi.org/10.1016/S0025-6196(12)62083-7)
3 Kloppel G. Classification and pathology of gastroenteropancreatic neuroendocrine neoplasms. Endocrine-Related Cancer 201118 (Supplement 1) S1-S16. (https://doi.org/10.1530/ERC-11-0013)

4 Cryer PE, Axelrod L, Grossman AB, Heller SR, Montori VM, Seaquist ER, Service FJ \& Endocrine Society. Evaluation and management of adult hypoglycemic disorders: an Endocrine Society Clinical Practice Guideline. Journal of Clinical Endocrinology and Metabolism 200994 709-728. (https://doi.org/10.1210/jc.2008-1410)

5 van Beek AP, Emous M, Laville M \& Tack J. Dumping syndrome after esophageal, gastric or bariatric surgery: pathophysiology, diagnosis, and management. Obesity Reviews 201718 68-85. (https://doi. org/10.1111/obr.12467)

6 Roldán Baños S, Ruiz de Angulo Martín D, Munítiz Ruiz V, Martinez de Haro L \& Parrilla Paricio P. Dumping syndrome with severe hypoglycaemia after nissen fundoplication in adults. Case report and literature review.. Endocrinología y Nutrición 201461 550-551. (https://doi.org/10.1016/j.endonu.2014.06.007)

7 Bernard B, Kline GA \& Service FJ. Hypoglycaemia following upper gastrointestinal surgery: case report and review of the literature. $B M C$ Gastroenterology 201010 77. (https://doi.org/10.1186/1471-230X-10-77)

8 Sundin A. Radiological and nuclear medicine imaging of gastroenteropancreatic neuroendocrine tumours. Best Practice and Research Clinical Gastroenterology 201226 803-818. (https://doi. org/10.1016/j.bpg.2012.12.004)

9 Tucker ON, Crotty PL \& Conlon KC. The management of insulinoma. British Journal of Surgery 200693 264-275. (https://doi. org/10.1002/bjs.5280)

10 Kartalis N, Mucelli RM \& Sundin, A. Recent developments in imaging of pancreatic neuroendocrine tumors. Annals of Gastroenterology 201528 193-202.

11 Sowa-Staszczak A, Trofimiuk-Muldner M, Stefanska A, Tomaszuk M, Buziak-Bereza M, Gilis-Januszewska A, Jabrocka-Hybel A, Głowa B, Małecki M, Bednarczuk T, et al. 99mTc labeled glucagon-like peptide-1-analogue (99mTc-GLP1) scintigraphy in the management of patients with occult insulinoma. PLoS ONE 201611 e0160714. (https://doi.org/10.1371/journal.pone.0160714)

12 Asa SL. Pancreatic endocrine tumors. Modern Pathology 201124 (Supplement 2) S66-S77. (https://doi.org/10.1038/ modpathol.2010.127)

13 Oberg K, Knigge U, Kwekkeboom D, Perren A \& Group EGW. Neuroendocrine gastro-entero-pancreatic tumors: ESMO Clinical Practice Guidelines for diagnosis, treatment and follow-up. Annals of Oncology 201223 (Supplement 7) vii124-vii130. (https://doi. org/10.1093/annonc/mds295)

14 Wei J, Liu X, Wu J, Xu W, Gao W, Jiang K, Zhang Z \& Miao Y. Diagnosis and surgical management of insulinomas in 33 consecutive patients at a single institution. Langenbeck's Archives of Surgery 2016 401 1019-1025. (https://doi.org/10.1007/s00423-016-1496-y)

15 Wu M, Wang H, Zhang X, Gao F, Liu P, Yu B, Qiu H, Huang G \& Tan X. Efficacy of laparoscopic ultrasonography in laparoscopic resection of insulinoma. Endoscopic Ultrasound 20176 149-155. (https://doi.org/10.4103/2303-9027.194703)

Received in final form 10 December 2017

Accepted 18 December 2017 